\title{
In Clinic Effectiveness of Heme Iron Polypeptide in Women with Iron Deficiency Anemia Nonresponsive or Noncompliant to Conventional Iron Supplements
}

\author{
Varsha Narayanan ${ }^{1 *}$ and Amit Bhargava ${ }^{2}$ \\ ${ }^{1}$ DGM Medical services, Lupin Ltd, India \\ ${ }^{2}$ VP Medical Services, Lupin Ltd, India
}

Submission: August 24, 2018; Published: September 14, 2018

*Corresponding author: Varsha Narayanan, DGM- Medical Services, Lupin Ltd, Laxmi towers 'C', BKC, Mumbai, India; Email: varshanarayanan@lupin.com

\begin{abstract}
Background: Iron deficiency anemia affects the quality of lifeand productivity of women, and in pregnancy can determine and affect maternal and fetal outcomes.Thoughoral iron supplementation is usually first line therapy due to convenience and cost, the conventional Nonheme iron oral supplements may present with gastrointestinal intolerability, poor compliance and limiting efficacythereby compromising patient benefit. Heme iron polypeptide (HIP) supplementation may provide an alternativeoption for oral iron therapy due to better efficacy and tolerabilityseen inrandomized clinical trials, therefore it would be relevant to observe if patients who have tried conventional iron supplements unsatisfactorily, can benefit from HIP in thereal-world setting.
\end{abstract}

Methodology: In this study, 270 womendiagnosed with Iron deficiency anemia, visiting gynecology clinicsand who were on conventional iron supplements, with poor gastrointestinal tolerance and compliance were evaluated fortheir Hemoglobin (Hb) and Serum Ferritin, and then treated with daily tablets of Heme Iron polypeptide (HIP) and followed up within a month for reassessment. Hemoglobin and Serum Ferritin assessed at start of HIP therapy (baseline) were repeated at the next follow up visit along with recording patients' tolerability to therapy.

Results: The rise in $\mathrm{Hb}$ was highly significant at the follow up visit in women who had baseline $\mathrm{Hb}$ indicating moderate and severe anemia (moderate group $\mathrm{Hb}$ rise in non-pregnant, pregnant: $1.15 \mathrm{~g} / \mathrm{dl}, 1.48 \mathrm{~g} / \mathrm{dl}$ and severe group $\mathrm{Hb}$ rise in non-pregnant, pregnant: $1.56 \mathrm{~g} / \mathrm{dl}, 2.75 \mathrm{~g} /$ $\mathrm{dl} ; \mathrm{P}<0.001$ ). There was also a significant decline in the number of patients with severe anemia, as well as those with low ferritin.The adverse event related discontinuation rate was $1 \%$ indicating a satisfactory tolerance and compliance to treatment.

Conclusion:This real-world study suggests that oral HIP therapy can be an effective treatment option in pregnant and non-pregnant women with Iron deficiency anemia due to gastrointestinal tolerability, compliance and efficacy in improving hematological parameters.

Keywords: Iron deficiency anemia; Heme iron polypeptide; Hemoglobin; Compliance; Gastrointestinal tolerability

Abbreviations: HIP: Heme iron polypeptide; Hb: Hemoglobin; IDA: Iron Deficiency Anemia; GI: Gastrointestinal

\section{Introduction}

Anemia affects almost2 billion people globally, with more than half thepregnant women and $>40 \%$ of non-pregnant women in developing countries being anemic[1,2]. In India this prevalence goes up to an alarming $89.6 \%-100 \%$ in pregnant women[3-5].

Irondeficiency is by far the most important cause of nutritional anemia worldwide as well as in developing countries like India[6]. Poverty, social practices and food habits contribute to Dietary Iron deficiencies, while poor bioavailability and tolerability of iron supplements pose therapeutic challenges. To add, parasitic infections, closely spaced pregnancies and heavy menstrual blood losses are some of the common causes contributing to high anemia prevalence in India[7].

The recommended iron intake in non-pregnant women is at least $12 \mathrm{mg}$ /day however not even $10 \%$ women in India meet this requirement[8].Anemia in pregnancy is dilutional in nature due to increasing total blood volume and red cell mass as well as increased demand from growing fetus,[9]Therefore pregnant women require a higher recommended iron intake in pregnancy of around $35 \mathrm{mg} /$ day[10]which is also far from met by dietary iron alone. So,in order to maintain hemoglobin levels,adequate, effective iron supplementation is essential for both maternal and 
fetal well-being in pregnant women as well as for the productivity and quality of life of non-pregnant women with IDA[12].

Dietary iron is contained in two forms namely non-heme and heme. While non-heme iron supplements have been conventionally prescribed, they are associated with poor gastrointestinal (GI) tolerability and constipation, require administration on empty stomach, have varying bioavailability due to different iron salts, and interact with certain drug and food items, all of which present as major therapeutic and compliance limitations which worsen even further in pregnancy[12,13].

Heme iron, available as heme iron polypeptide (HIP) tablets for oral supplementation represents a therapeutic option for iron supplementation with lower dose requirement due to higher bioavailability and gastrointestinal tolerability[14].

Our real world in clinic data captureevaluates the effectiveness and tolerability of oral Heme Iron Polypeptide (HIP) supplementation in both non-pregnant and pregnant women with Iron deficiency anemia (IDA) who have not experienced satisfaction in therapy with conventional iron supplementson account of inability in maintaining compliance or GI tolerability.

\section{Methodology}

270female patients with an in-clinic diagnosis of iron deficiency anemia (IDA) at gynecology clinics across India and who were on conventional iron supplements, with poor gastrointestinal tolerance and compliance,were made to discontinue their current iron supplements and re-evaluated for Hemoglobin $(\mathrm{Hb})$ and serum Ferritin which were taken as baseline values. Based on the baseline $\mathrm{Hb}$ values patients were grouped as mild, moderate and severe anemia based onWHO cut off reference values in pregnant and non-pregnant women[15]. Patients were also classified to have low, or normal ferritin levels based on WHO cut off values[16].Thereafter these patients with IDAwere given 1-2 tablets of $12 \mathrm{mg}$ Heme Iron polypeptide (HIP) daily with or without meals and called for first follow up assessment visit within a month.

The patients were assessed at follow up for mean rise in the blood parameters recorded at baseline. Primary end points were mean rise in Hemoglobin and tolerability with HIP. Secondary end points included overall reduction in severity of anemia and increase in serum ferritin.

Ethical principles, patient consent and confidentiality of identity were adhered to while capturing the all patient data.

\section{Result}

Out of the 270 female patients who were prescribed HIP daily, majority ( $85 \%$ ) patients were in the $20-40$ years age group with23pregnant women. More than $50 \%$ patients complained of fatigue or weakness as the predominant symptom. $30 \%$ of the non-pregnant patient group had a previous history of pregnancy and a similar number in this groupalso gave history of heavy menstrual bleed. Gastrointestinal intolerability due to nausea, vomiting, constipation as well as no perceived improvement in symptoms were the reasons for poor compliance and unsatisfactory response to conventional iron supplements in these patients.

The mean rise in hemoglobin at first follow up visit is shown in Figure 1\&2. At baseline, the pregnant women were all in moderate or severe anemia group and the rise in $\mathrm{Hb}$ at first follow up visit was $1.48 \mathrm{~g} / \mathrm{dl}$ and $2.75 \mathrm{~g} / \mathrm{dl}$ in the moderate and severe anemia group respectively ( $\mathrm{P}=0.0001$ and 0.0076 vs baseline). In non-pregnant women, the rise in $\mathrm{Hb}$ at first follow up visit was $1.02 \mathrm{~g} / \mathrm{dl}, 1.15 \mathrm{~g} / \mathrm{dl}$ and $1.56 \mathrm{~g} / \mathrm{dl}$ in the mild, moderate and severe anemia groups respectively $(\mathrm{P}<0.0001$ vs baseline for moderate and severe anemia groups).

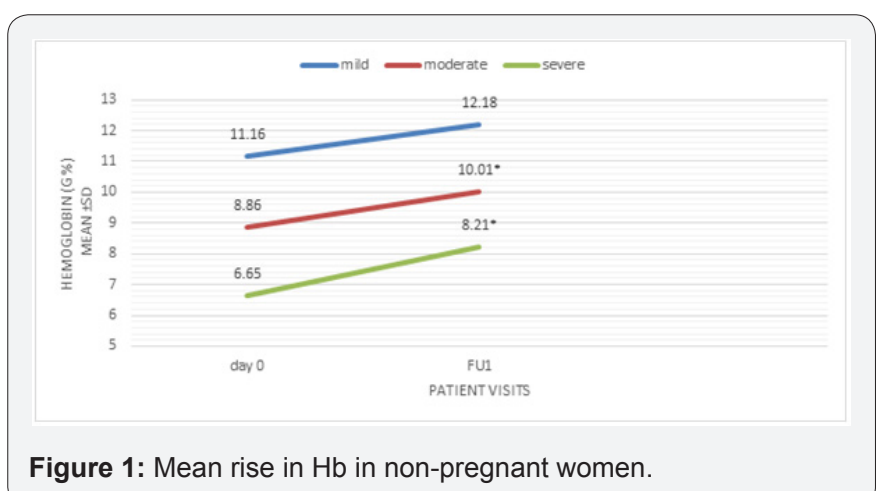

${ }^{*} \mathrm{P}<0.0001 \mathrm{FU} 1$ (1st follow up visit) vs day 0 (baseline).

WHO cut offs $\mathrm{Hb}$ g/dl: (non-pregnant) Normal: $>12$, Anemia: Mild $<11.0-11.9$, Moderate 8.0-10.9, Severe $<8$.

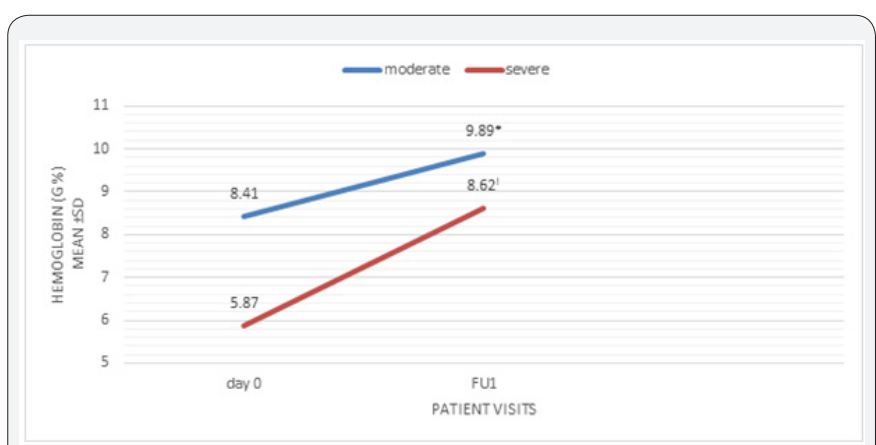

Figure 2: Mean rise in $\mathrm{Hb}$ in Pregnant women.

${ }^{*} \mathrm{P}=0.0001$ FU1 (1st follow up visit) vs day 0 (baseline); $\mathrm{P}=0.0076$ FU1 vs day 0 (baseline)

WHO cut offs Hb g/dl: (pregnant) Normal: $>12$, Anemia: Mild $<10.0-$ 10.9, Moderate 7.0-9.9, Severe $<7$

The percentage of patients who had severe anemia, decreased significantly from $40 \%$ to $13 \%$ and from $19 \%$ to nil at the first follow up visit in non-pregnant and pregnant patients respectively $(\mathrm{P}<0.05)$ (Figure 3$)$.

Average serum ferritin was seen to increase from $94.87 \mathrm{ug} /$ $\mathrm{dl}$ to $109.6 \mathrm{ug} / \mathrm{dl}$, and this absolute increase was not found to be significant. However, the percentage of patients with low ferritin 
levels decreased from $20 \%$ and $37.5 \%$ to $1.2 \%$ and nil in nonpregnant and pregnant women at first follow up $(\mathrm{P}<0.0001)$ (Figure 3). Adverse events mainly gastrointestinal disturbance, which included nausea, abdominal cramps and constipation was seen in less than $10 \%$ patients with 3 patients (1\%) having to discontinue treatment.

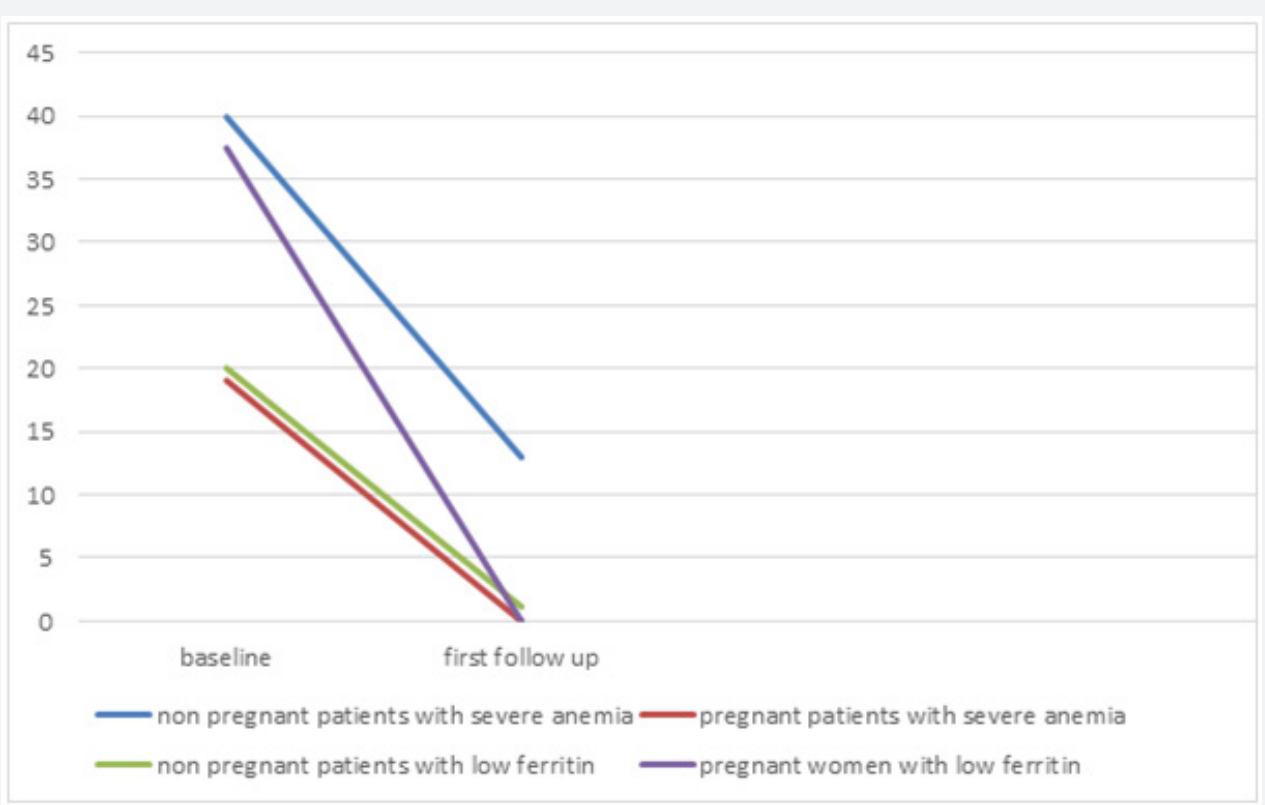

Figure 3: Proportion of patients (\%) with severe anemia and low ferritin.

WHO cut offs for severe anemia Hb g/dl[15]: (non-pregnant:8; pregnant 7); cut off for low ferritin/depleted iron stores: $15 \mathrm{ug} / \mathrm{dl})[16]$.

\section{Discussion}

HIP is a form of iron supplementation with higher bioavailability and gastrointestinaltolerability due to higher solubility at low pH and enhanced binding to heme receptor thereby improving its cellular entry[17-19].A 10 times higher bioavailability of heme iron compared to non-heme iron, have been reported in volunteerstudies[20].In a study where HIP, ferrous fumarate, and placebo was administered with breakfast to patients, HIP led to increased iron absorption even with meal as compared to ferrous fumarate and placebo $(\mathrm{p}<0.03$ and $\mathrm{p}<$ 0.02 respectively) without any side effects[20].

HIP has advantages over non-heme iron supplements as substances in food like tannins and phytates (present commonly in Indian diets) and chelators like desferrioxamine do not reduce its bio- availability unlike as seen with non-heme iron supplements[21]. A study on HIP supplementation observed significant increase in serum iron levels in subjects with low initial serum iron levels $(<80 \mu \mathrm{g} / \mathrm{dl})$ compared to those with normal initial iron levels, suggesting that continual supplementation of heme-iron may not lead to iron overload and HIP absorption $s$ regulated by body iron levels and heme receptor[18].

Suzuki et al evaluated the efficacy of Heme Iron Polypeptide $3 \mathrm{mg}$ in pregnancy and in females with bleeding disorderand demonstrated gradual rise in $\mathrm{Hb}$ over a 14-week period with most patients reaching an $\mathrm{Hb}$ above $11 \mathrm{gm} / \mathrm{dl}$ with twice daily regime[22].Authors have suggested that average dose of 6.5 mg elemental iron as HIP or maximum dose of $9 \mathrm{mg}$ elemental iron as HIP is sufficient and well tolerated to improve anemia in pregnant women when administered in the third trimester.

A recent study comparing oral HIP and intravenous iron saccharate complex in pregnant women with iron deficiency anemia $(\mathrm{Hb}<10 \mathrm{gm} / \mathrm{dL})$ showed $\mathrm{Hb}$ increase from $8.5+/-3.5$ to $11.3+/-1.3 \mathrm{~g} / \mathrm{dl}$ in HIP treatment group, and an $\mathrm{Hb}$ increase from $8.7+/-2.5$ to $11.7+-0.9 \mathrm{~g} / \mathrm{dl}$ in IV iron treatment group accompanied by increased ferritin in both groups at end of three months (no significant differences between groups for change in $\mathrm{Hb}$ or ferritin levels)[23]. GI upset was reported in $1.6 \%$ of patients receiving oral HIP. This suggested oral HIP as an effective and tolerable treatment which can be considered as an alternative to intravenous iron saccharate complex for iron deficiency anemia of pregnancy.

In two different studies, Young et al evaluated intrinsically radiolabeled Fe-heme and Fe non-heme (ferrous sulphate) given to pregnant women and non-pregnant women. In both pregnant and non-pregnant women, blood samples obtained 2-week post dosing to assess iron status indicators, serum hepcidin and iron utilization based on RBC incorporation of iron isotopes showed that heme-iron utilization was significantly higher relative to utilization of ferrous sulphate among both pregnant $(\mathrm{p}=0.04)$ and non-pregnant women $(\mathrm{p}<0.0001)[24]$.While 
non-heme iron utilization is inversely related to serum hepcidin (as evidenced by women with undetectable levels of serum hepcidin having significantly greater non-heme iron utilization, and a $24 \%$ variation in non-heme iron utilization seen in healthy pregnant and non-pregnant participants due to hepcidin levels), heme iron utilization is not seen to be significantly influenced by iron stores or serum hepcidin.The second study found that in pregnant women in third trimester, maternally absorbed $\mathrm{Fe}$ Heme tracer present in the neonates was significantly greater compared to the Fe-Non-heme tracer ( $p=0.02$ ).Also, fetal transfer was inversely associated with maternal $\mathrm{Hb}$ and ferritin levels, whereas total body iron significantly correlated with heme iron transfer for heme iron tracer, suggesting favourable transport across placenta[25].

Compliance to Heme Iron therapy due to low GI side effects has been a major reason for the efficacy results in clinical studies. A study in pregnant and post-partum women noted a compliance of $<50 \%$ in $4 \%$ of the heme-iron group, $12 \%$ of the non-heme iron group and $5 \%$ of the placebo group. Women who failed to maintain an acceptable hematologic status were $10 \%$, $20 \%$ and $45 \%$ in heme iron, non-heme iron and placebo group respectively[26]. Hb levels improvedsignificantly only amongst strictly compliant pregnant women and anemiawas significantly associated with noncompliance with iron supplementation has also been demonstrated with anadjusted OR being 6.19 (95\% CI 2.55 - 15.02, $\mathrm{p}<0.0001$ )[27].

Our in-clinic effectiveness study findings further lend credence to results of earlier studies with HIP.The average early rise in $\mathrm{Hb}$ at first follow up visit itself was significant in both pregnant and non-pregnant women with IDA andas seen in our study was $1.02 \mathrm{~g} / \mathrm{dl}, 1.15 \mathrm{~g} / \mathrm{dl}$ and $1.56 \mathrm{~g} / \mathrm{dl}$, in the baseline mild, moderate and severe anemia non-pregnant patient groups and $1.48 \mathrm{~g} / \mathrm{dl}$ and $2.75 \mathrm{~g} / \mathrm{dl}$ in the baseline moderate and severe anemiapregnant patient groups.Hb rise was seen to be more in patients with lower baseline $\mathrm{Hb}$, as has also been seen in other studies. There was also a significant decline in the number of patients with severe anemia, as well as in percentage patients with low ferritin. The tolerability of HIP in our study was similar to other published studies and was better than the conventional iron supplements that the patients were on previously, with a very low treatment discontinuation rate and satisfactory patient compliance.

\section{Conclusion}

Oral HIP therapy can be an effective treatment and an option of choice for both pregnant and non- pregnant women with Iron deficiency anemia especially in those patients who are noncompliant, non-responsive or poorly tolerating conventional non-heme iron supplements. HIP with its better absorption, GI tolerability and efficacy in improving hematological parameters can add to the armamentarium of IDA therapy.Further studies comparing various heme and non-heme oral iron supplements as well as also studying symptomatic improvement in a varied range of patients can further add insights in effective management of iron deficiency anemia.

\section{References}

1. De Maeyer EM, Adiels TM (1985) The prevalence of anaemia in the world. World Health Stat Q 38(3): 302-316.

2. World Health Organization (WHO) (1992) The Prevalence of Anaemia in Women: A Tabulation of Available information. Geneva, Switzerland: WHO.

3. Prevalence of Anemia among Pregnant Women (\%). World bank 2011.

4. Bora R, Sable C, Wolfson J, Boro K, Rao R (2014) Prevalence of Anemia in Pregnant Women and Its Effect on Neonatal Outcomes in Northeast India. The Journal of Maternal -Fetal \& Neonatal Medicine 27: 887-891.

5. Vemulapalli B, Rao KK (2014) Prevalence of Anemia among Pregnant Women of Rural community in Vizianagram North Costal Andhra Pradesh, India. AJMS 5(2): 21-25.

6. DeMaeyer EM, Dallman P, Gurney JM, Hallberg L, Sood SK, et al. (1989) Preventing and controlling Iron Deficiency Anaemia Through Primary Health Care. Geneva: WHO.

7. Bharati P, Som S, Chakrabarty S, Bharati S, Pal M (2008) Prevalence of Anemia and Its Determinants among Nonpregnant and Pregnant Women in India. Asia Pac J Public Health 20(4): 347-359.

8. Milman N (2006) Iron and Pregnancy-A Delicate Balance. Ann Hematol 85: 559-565.

9. Chandra S, Tripathi AK, Mishra S, Amzarul M, Vaish AK (2012) Physiological Changes in Hematological Parameters during Pregnancy. Indian J Hematol Blood Transfus 28(3): 144-146.

10. Indian Council for Medical Research (2011) Dietary Guidelines for Indians-A Manual. $2^{\text {nd }}$ Edition, National Institute of Nutrition, Hyderabad, India, 89.

11. EFSA Panel on Food Additives and Nutrient Sources added to Food (ANS) (2010) Scientific Opinion on the Safety of Iron (Blood Peptonates) for the Proposed Uses as a Source of Iron Added for Nutritional Purposes to Foods for the General Population, Including Food Supplements. EFSA Journal 8: 1585.

12. Pavord S, Myers B, Robinson S, Allard S, Strong J, et al. (2012) UK Guidelines on the Management of Iron Deficiency in Pregnancy. British Journal of Hematology 156: 588-600.

13. Johnson-Wimbley TD, Graham DY (2011) Diagnosis and Management of Iron Deficiency Anemia in the 21st Century. Therap Adv Gastroenterol 4(3): 177-184.

14. Hoppe M, Brun B, Larsson MP, Moraeus L, Hulthen L (2013) Heme IronBased Dietary Intervention for Improvement of Iron Status in Young Women. Nutrition 29(1): 89-95.

15. WHO (2011) Haemoglobin concentrations for the diagnosis of anaemia and assessment of severity.

16. WHO (2011) Serum ferritin concentrations for the assessment of iron status and iron deficiency in populations.

17. Nagaraju SP, Cohn A, Akbari A, Davis JL, Zimmerman DL (2013) Heme Iron Polypeptide for the Treatment of Iron Deficiency Anemia in NonDialysis Chronic Kidney Disease Patients: A Randomized Controlled Trial. BMC Nephrology 14: 64.

18. Nam T, Shim JY, Kim B, Rah SY, Park K, et al. (2006) Clinical Study on the Iron Absorption from Heme-Iron Polypeptide and Nonheme-Iron. Nutritional Sciences 9: 295-300.

19. Nissenson AR, Charytan C (2003) Controversies in Iron Management. Kidney International 64: S64-S71. 
20. Seligman PA, Moore GM, Schleicher RB (2000) Clinical Studies of HIP: An Oral-Iron Product. Nutrition Research 20(9): 1279-1286.

21. Kalaivani K (2009) Prevalence \& Consequences of Anemia in Pregnancy. Indian J Med Res 130(5): 627-633.

22. Suzuki S, Ito H, Kiyokawa H (1990) The Effect of Biolron-150 on Pregnant Women and Women with Females Associated Bleeding Disorders. The World of Obstetrics and Gynecology 42: 33-42.

23. Abdelazimn IA, Abu-Faza M, Elbiaa AAM, Othman HS, Alsharif DA, et al. (2017) Heme Iron Polypeptide (Proferrin®-ES) versus Iron Saccharate Complex (Ferrosac) for Treatment of Iron Deficiency Anemia during Pregnancy. Acta Medica International 4(1): 55-60.

24. Young MF, Griffin I, Pressman E, McIntyre AW, Cooper E, et al. (2010) Utilization of Iron from an Animal-Based Iron Source Is Greater than
That of Ferrous Sulfate in Pregnant and Nonpregnant Women. J Nutr 140(12): 2162-2166.

25. Young MF, Griffin I, Pressman E, McIntyre AW, Cooper E, et al. (2012) Maternal Hepcidin Is Associated with Placental Transfer of Iron Derived from Dietary and Non-Sources. J Nutr. 142(1): 33-39.

26. Eskeland B, Malterud K, Ulvik RJ, Hunskaar S (1997) Iron Supplementation in Pregnancy: Is Less Enough? A Randomized, Placebo Controlled Trial of Low Dose Iron Supplementation with and without Iron. Acta Obstet Gynecol Scand 76(90): 822-828.

27. Habib F, Habib ZAE, Alenazy M, Nooh R (2009) Compliance to Iron Supplementation during Pregnancy. J Obstet Gynaecol 29(6): 487-492.

\begin{tabular}{l} 
Your next submission with Juniper Publishers \\
will reach you the below assets \\
- Quality Editorial service \\
- Swift Peer Review \\
- Reprints availability \\
- E-prints Service \\
- Manuscript Podcast for convenient understanding \\
- Global attainment for your research \\
- Manuscript accessibility in different formats \\
( Pdf, E-pub, Full Text, Audio) \\
- Unceasing customer service \\
Track the below URL for one-step submission \\
https://juniperpublishers.com/online-submission.php \\
\hline
\end{tabular}

